\title{
XVIII.
}

\section{Ueber Knochentuberculose.}

\author{
Von \\ Dr. Karl Jaffé \\ in Hamburg.
}

In dem ${ }_{n}$ Rück- und Vorblick", mit dem C. Hueter und Luicke den XI. Band dieser Zeitsehrift am 18. December 1878 eröffneten, beklagten sich die Verfasser darüber, dass die chirurgische Literatur der letzten 10 Jahre über die Tubereulosefrage nsich so gründlich ausschweigt." Selten wohl ist ein ähnlicher Wunsch rascher erhört worden, als der in den genannten Worten angedeutete. Gerade die letzten 4 Jahre haben, speciell auch von ehirurgischer Seite, eine ansehnliche Fülle von Tuberculosearbeiten zu Tage gefördert, und unsere heutigen Anschauungen über das Wesen und die Bedeutung dieser vielgestaltigen Krankheit, die gerade dureh die grosse Mannigfaltigkeit ihrer Erscheinung ihren Bearbeitern stets grosse Schwierigkeiten bereitete, sind speciell durch experimentelle Arbeiten der jüngsten Zeit so erweitert und gegen früher verändert worden, wie kaum bei irgend einer anderen Krankheit.

Wir stehen heute inmitten des Kampfes über die bacilläre Natur der Tubereulose, und es ist schwer abzusehen, wann und wie eine Einigung der widersprechenden Ansichten wird erzielt werden können. Es ist kaum ein Jahr her, dass $\mathrm{K}_{0} \mathrm{ch}$ durch die Entdeckung des Tuberkelbacillus ein neues Licht über die Aetiologie unserer Krankheit zu verbreiten schien. Aber schon mehren sich täglich die Stimmen, welche seine Schlussfolgerungen angreifen, und man kann heute nicht mehr tibersehen, wie viel von seiner ganzen Entdeckung Bestand haben wird. In einem solchen Kampfe, der alles miihsam bisher Errungene der Vernichtung preisgiebt, und wo mit einem Federstrich oft die Arbeiten von Generationen annullirt werden, ist es Pflicht, zu retten, was gerettet zu werden verdient, damit nicht der gesicherte Bestand ebenfalls ein Opfer der allgemeinen Zerstörung 
wird. Gerade die Chirurgie hat solche sichere Errungenschaften auf dem Gebiet der Tubereulosefrage in den letzten Jahren aufzuweisen, und es ist speciell die Tuberculose der Knochen und Gelenke, für deren Verständnis wir vieles gewonnen haben. Man wusste zwar schon lange, dass Tuberkel auf der Synovialis der Gelenke und im Knochenmark vorkommen können; auch batte Köster [17] ${ }^{1}$ ) bereits ihr Vorkommen in fungösen Gelenken und Friedländer (10) ihre anatomische Identität mit den Knötchen der gewöhnlichen Tuberculose gelehrt. Dass aber beide Erkrankungen (Knochen- und innere Tuberculose) in ihrem Wesen und ibrer klinischen Bedeutung nur verschiedene Formen eines und desselben Krankheitsprocesses sind, der bald local im Knochen beginnt und sich generalisirt, bald umgekehrt bei allgemeiner Infection auch den Knochen ergreift, bald aber auch local beginnt und local beschränkt bleiben kann, dieses auch praktisch hochwichtige Ergebnis verdanken wir einer Reihe von Arbeiten aus den letzten Jahren, unter denen ich als die wichtigsten nur diejenigen von $\mathrm{K} \ddot{\mathrm{nig}}(21-25)$, Volkmann $(42,43)$, Mü ch (28), C. und H. Hueter $(13,15,16)$, Schüller $(35,36$, $36 a)$, Boegehold (5) und Baumgarten (1) nennen möchte. Wir verstehen also unter Knochen- resp. Gelenktuberculose alle diejenigen Processe, die man früher als nscrophulöse oder tuberculöse Caries, fungöse Gelenkentzündung, Tumor albus “ u. s. w. bezeichnet hatte, ohne damit irgend etwas über ihre mikroskopische Zusammensetzung oder Aetiologie präjudiciren zu wollen.

Für uns ist die Knochentuberculose keine ätiologische, sondern nur eine klinische Einheit, und es würde für jetzt wenigstens nur wieder neue Verwirrung stiften, wenn man etwa von dem Nachweis von Bacillen die Diagnose "Knochentuberculose" abhängig machen wollte. Auch die von Virchow immer urgirte Trennung zwischen käsiger und tuberculöser Substanz lässt sich, für die uns hier beschäftjgenden Processe wenigstens, nicht durchführen. Es ist darum noch nicht nöthig, wie Talma (39) wïnschte, den Namen „Tuberculose" ganz zu streichen. So wenig der anatomische Begriff" "Tuberkel “ von der Riesenzelle abhängig gemacht zu werden braucht ${ }^{2}$ ), die doch ein fast constanter Begleiter desselben ist, so wenig gehört zum klinischen Krankheitsbild der Knochentuberculose nothwendig der Nachweis des "gefässlosen Knötchens", das längst in Conglomeratknoten und käsiges Gewebe aufgegangen sein kann.

1) Die eingeklammerten Zahlen hinter den Autoren beziehen sich auf das Literaturverzeichniss am Schluss.

2) Vgl. Heiberg, Die Tuberculose in ihrer anatomischen Beziehung. S. 14. 
Fragen wir nun von diesem Standpunkt aus, wie es mit unserer Kenntnis von der Häufigkeit des Vorkommens der Knochentuberculose steht, so scheint mir gerade hier noch manche Luicke zu existiren. So viel mir bekannt, ist seit dem Jahr 1870, wo Bill roth und Menzel (4) an einem Sectionsmaterial von 1996 Fällen von Knochencaries eine tabellarische Zusammenstellung gemacht Laben, keine grössere Arbeit über diesen Gegenstand erschienen. Es schien mir deshalb nicht unangemessen, das Material des hiesigen allgemeinen Krankenhauses in dieser Hinsicht zu verwerthen. ${ }^{1}$ ) Ich habe nur diejenigen Fälle der letzten 2 Jahre - es sind im Ganzen 317 - benutzt, die ich entweder selbst als Assistent bis zum Austritt aus dem Spital beobachten, oder deren Operation resp. Section ich anwohmen konnte, und über welehe mehr oder weniger ausführliche Aufzeichnungen vorlagen. Bin ich in Bezug auf die Gesammtzahl Billroth und Menzel gegenüber im Nachtheil, so habe ich doch daftir nicht unerhebliche Vortheile ihnen voraus, die wohl geeignet sein dürften, die geringere Summe der Fälle hinreichend aufzuwiegen. Als solche betrachte ich erstlich die Thatsache, dass ich über geheilte, ungeheilte und gestorbene Fälle berichten kann, während die genannten Autoren nur Sectionsresultate benutzten; ferner fehlen ihnen die Kinder ganz, die doch einen erheblichen Beitrag zur Knochentuberculose liefern. Endlich standen mir fast ausschliesslich selbst beobachtete Fälle zur Verfigung, während Jene nur über fremdes Material verfügten, worunter stets die Sicherheit in den Angaben zu leiden hat, wie Jeder zugeben wird, der einmal genöthigt gewesen ist, fremde Krankengeschichten für bestimmte Gesichtspunkte verwerthen zu müssen. Ich konnte somit eine Anzahl yon Rubriken zur Vergleichung aufstellen, die das Verhältnis der mit oder ohne Operation Genesenen und Aehnliches beleuchten sollen, wobei sich noch manche andere Beziehung zu Fragen der praktischen Chirurgie ergeben hat.

Ausser den rein ostalen Formen der Tuberculose sind auch eine Anzahl tuberculöser Gelenkaffectionen mit angezogen worden, die mit den ersteren in vieler Beziehung ubereinstimmen. Hierher gehören erstens alle secundären Gelenktuberculosen, wo der primäre Herd im Knochen seinen Sitz hat; ferner jene äusserst selten und fast nur bei Erwachsenen vorkommenden Formen primärer Synovialistuber-

1) Für die Erlaubnis zur Veröffentlichung dieses Materials sage ich meinem verehrten ehemaligen Chef, Herrn Dr. Max Schede, auch öffentlich meinen verbindlichsten Dank. 
culose, welche den Knochen erst spät in Mitleidenschaft ziehen; drittens endlich eine Anzahl leichterer fungöser Gelenkerkrankungen, welche durch ihr Vorkommen an scrophulösen Individuen, häufig noch gleichzeitig mit schwereren Formen an anderen Gelenken combinirt, uber ihre Natur nicht im Dunkeln liessen, trotzdem sie nicht zur Operation gelangten und einer expectativen oder subcutan angewendeten Therapie wichen.

Die Anordnung des Stoffes ergibt sich aus den Ueberschriften der einzelnen Abschnitte, in deren Eintheilung ich zum Theil der citirten Arbeit von Billroth und Menzel gefolgt bin.

\section{Principien bei der Aufstellung der Fundamentaltabelle.}

Bei der Anfertigung der beigefigten (S. 437) Fundamentaltabelle, die einen Ueberblick über das verwerthete Material gewähren soll und die allen später folgenden Ergebnissen zu Grunde gelegt worden ist, waren folgende Gesichtspunkte die leitenden.

Die verticalen Rubriken sind streng topographisch geordnet und umfassen die drei Hauptabschnitte für die Körpergegenden, nämlich

Kopf und Stamm,

Obere Extremität,

Untere Extremität.

Jede derselben zerfällt wieder in 3, resp. 6, resp. 7 Unterabthejlungen, welche die einzelnen Knochengruppen einsçhliessen. Die 6 Hauptgelenke des Körpers (Schulter-, Ellenbogen-, Hand-, Hüft-, Knie- und Fussgelenk) sind, trotzdem viele Fälle primär ostaler (Tuberculose dabei sind, besonders rubricirt, weil man auch in solchen Fällen noch immer von einer Gelenkerkrankung redet, selbst wenn die Kapsel wenig oder gar nicht ergriffen ist. Von allen Knochen fehlt nur der Radius, der iiberhaupt am seltensten tuberculös zu erkranken scheint. Auch in Billroth's und Menzel's 1996 Cariesfällen ist er nur 2 mal notirt. Bei gleichzeitiger Erkrankung mehrerer Knochen ist der Fall in die Rubrik derjenigen Knochen eingereiht, welche die primär erkrankten waren; ausserdem sind aber alle Fälle multipler Knochentuberculose noch einmal besonders verzeichnet. Die in der letzten verticalen Columne aufgefïhrten "Resultate" geben die Summe der einzelnen in der horizontalen Rubrik befindlichen Fälle in Zahl und Procenten; bei den Hauptrubriken ist noch besonders die Summe für die drei Hauptabschnitte des Körpers wiedergegeben.

Die in den wichtigeren Rubriken vorkommenden kleinen Zahlen, 
welche unter den grossen Hauptzahlen stehen, bezeichnen für jede einzelne Knochengruppe das Procentverhältnis ihres Vorkommens, and zwar das procentische Verhältnis der Summe aller Fälle, wie sie in jeder Gruppe vorgekommen sind. So sind z. B. von 82 Spondylitisfällen 56 geheilt ${ }^{1}$ ), also 68 Proc., was ein Blick auf die betreffende Rubrik der Tabelle sofort ergibt.

Als nohne operativen Eingriff Geheilte" sind die Fälle mit aufgenommen, wo durch Gyps- und Extensionsverbände, Umschläge, ableitende Mittel, diätetische Behandlung u. s. w. eine so erhebliche Besserung herbeigeführt werden konnte, dass die Patienten ohne wesentliche Krankheitserscheinungen nach Hause entlassen werden konnten. Hierher gehören eine grosse Anzahl Spondylitiden, ferner besonders manche Formen beginnender Coxitis und Tumor albus genu, die dureh ihr Auftreten bei scropbulösen oder tuberculösen Individuen ihre tuberculöse Natur documentirten und bei rechtzeitigem richtigem Régime gar nicht so selten ansheilen können. Dieselbe Auswahl wurde unter den mit intraarticulären resp. - ostalen Injectionen Behandelten getroffen; es sind nur solche Fälle aufgenommen, wo die Diagnose der tuberculösen Erkrankung nicht zweifelhaft sein konnte. Unter Kranken, die durch "Nekrotomie" geheilt sind, sind natürlich keine typipischen Nekrosen zu verstehen; es handelt sich dabei immer um käsige Sequester, zu deren Entfernung kleinere oder grössere Partien der Sequesterlade fortgemeisselt werden mussten.

Die Alterstabelle schreitet von $10 \mathrm{z} n 10$ Jahren fort; für das besonders interessirende erste Jabrzehnt ist noch eine eigene Curve gezeichnet, die gehörigen Orts folgen wird.

1) Um nahe liegenden Einwänden a limine vorzubeugen, bemerke ich, dass in der vorliegenden Arbeit unter "g ebeil t" alle diejenigen Fälle aufgeführt sind, bei denen objectiv der ursprüngliche Krankheitsprocess als abgelaufen erschien und subjectiv die Kranken sich soweit wohl fühlten, dass sie ihre Häuslichkeit wieder aufsuchen konnten. Eine andere Frage, die ausserbalb des Rabmens dieser Arbeit lag, ist ja, wieweit diese "Heilungen" Bestand haben? Die König'schen Angaben (23), die Resectionen betreffend, haben hier manche Hlusion zerstört. Ich selbst habe bei der Spondylitis, deren abnorm hohe Heilungsziffer gerechtes Bedenken hervorrufen konnte, Nachforschungen über das fernere Schicksal der als "geheilt" Entlassenen angestellt, und es ist mir dies in 25 Fällen gelungen. Von diesen sind denn auch $5=20$ Proc. bereits gestorben; $3=12$ Proc. sind noch bettlägerig und leidend; die übrigen $17=68$ Proc. dagegen befinden sich alle in guter Gesundheit; die betreffenden Kinder sind in der Schule thatig, die Erwachsenen gehen ihren Geschäften nach. Ich gestehe gern zu, dass ich von dieser hohen Ziffer der anhaltenden Heilung freudig überrascht war, treibe den Skepticismus nun aber auch nicht so weit, bei diesen wohl constatirten Fällen an dem ferneren Bestand der Heilung zweifeln zu wollen. 


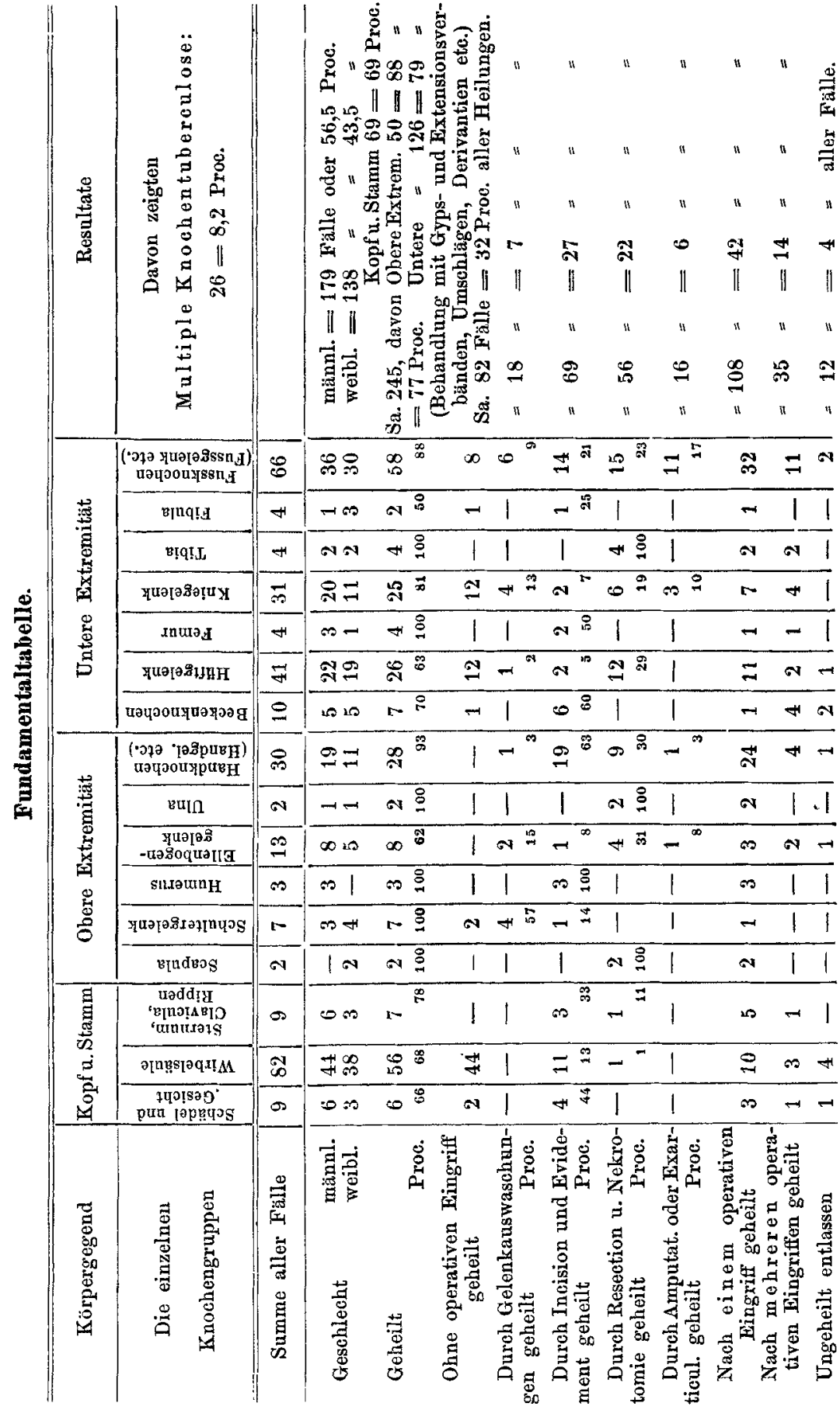




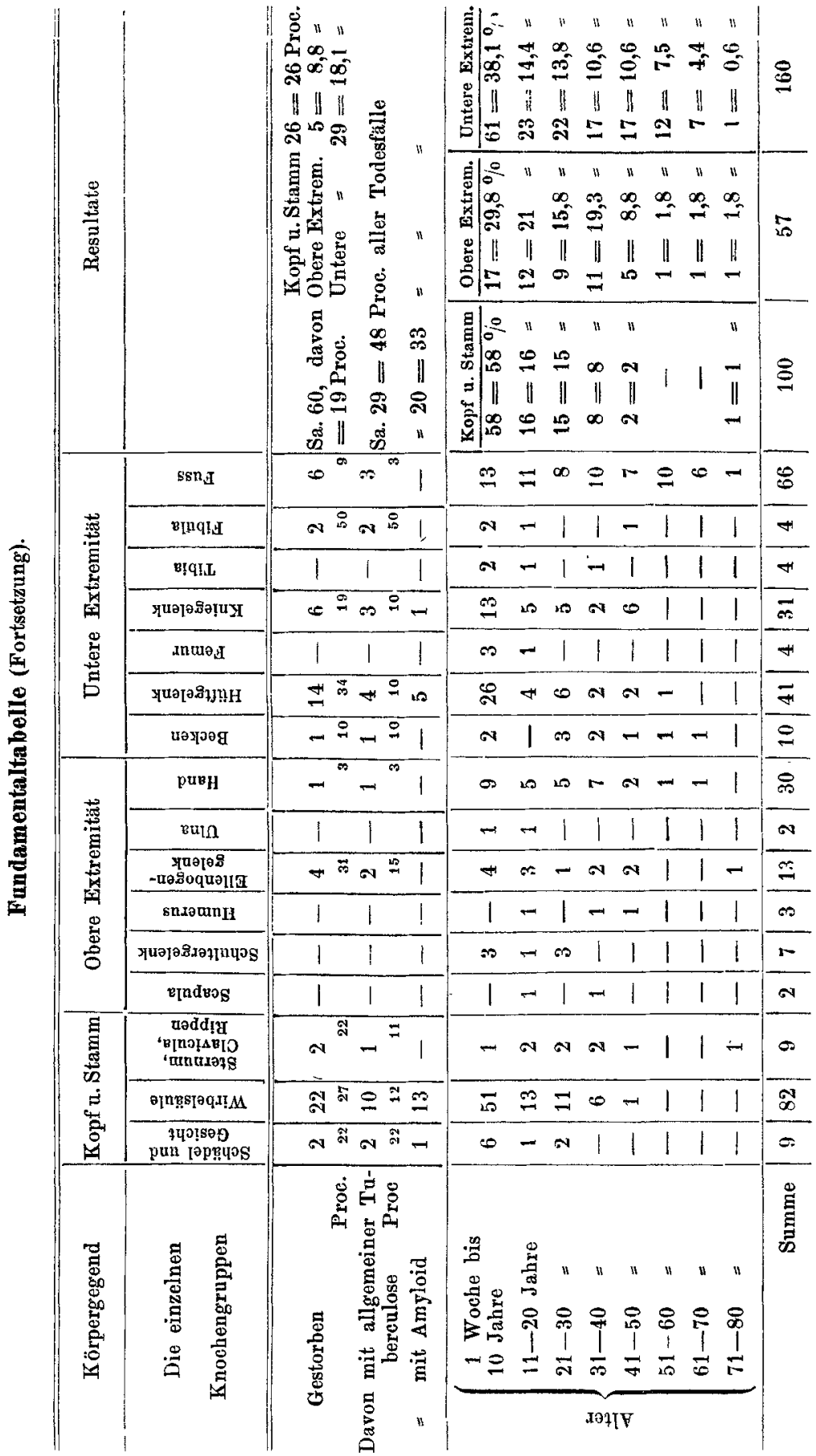


Die Sectionsergebnisse sind aus den sehr sorgfältig geführten Protokollen des Prosectors des Allgemeinen Krankenhanses, Herrn Dr. E. Fränkel, extrahirt; wo ein besonderes Sectionsprotokoll der Krankengeschichte beilag, ist dasselbe mit den ersteren verglichen und controlirt worden.

Endlich braucht wohl kaum versichert zu werden, dass bei der Aufstellung und Umrechnung der Zahlen die möglicbste Sorgfalt und Genauigkeit verwandt worden ist.

\section{Häufigkeit der Knochentuberculose nach den Körpergegenden.}

Ein Blick auf die unten angefïgte Curve 1, die in den Abscissen die einzelnen Knochengruppen enthält, während die Ordinaten die Curve t.

$\frac{\begin{array}{c}\text { Kopf und } \\ \text { Stamm }\end{array} \mid \text { Obere Extremität }}{\text { Untere Extremität }}$

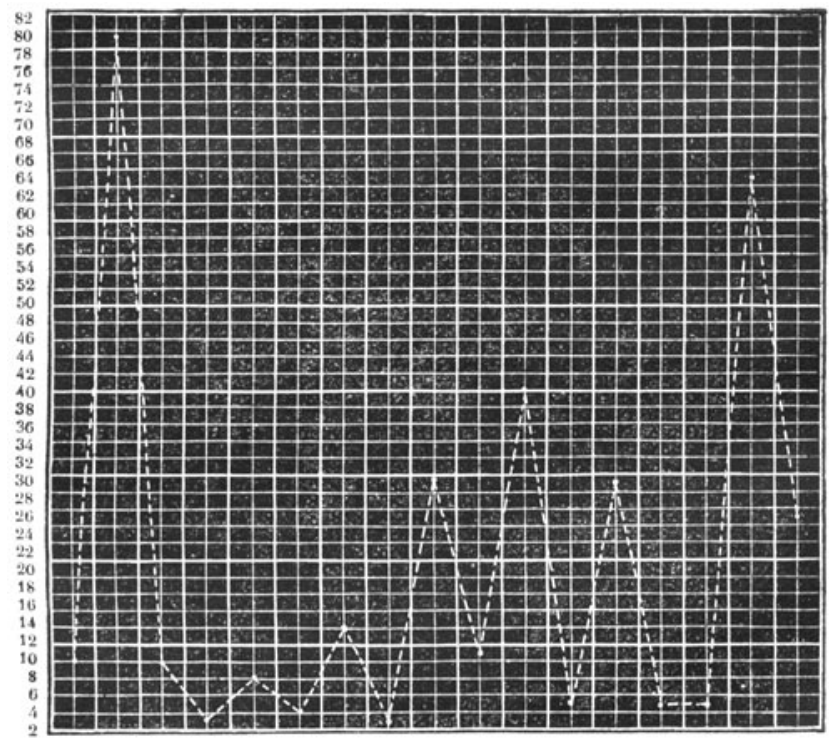

Graphische Darstellung der Häufigkeit tuberculöser Erkrankungen in den verschiedenen Kuochengruppen bei 317 Fällen von Knochentuberculose. Die Curve zeigt die absolute Zahl der vorgekommenen Fälle.

absolute Zahl der beobachteten Fälle darstellen, gibt sofort eine Uebersicht über die Häufigkeit des Vorkommens der Knochentuber- 
culose an den versehiedenen Körpergegenden. Die daraus sich ergebende Reihenfolge ist demnach folgende:

1. Wirbelsäule . . mit 82 Fällen $=26$ Proc.

2. Fussknochen . . $=66=21=$

3. Hüftgelenk . . . $=41=-13=$

4. Kniegelenk . . . $=31=-10=$

5. Handknochen . $=30==9=$

6. Ellenbogengelenk $\cdot=13==4=$

7. Beckenknochen . . $=10=-3=$

8. Schädel und Gesicht $=9==3=$

9. Sternum, Olavicula $\}=9=-3=$

10. Schultergelenk: : $=7==2=$

11. Femur

12. Tibia $\}$. . . mit je $4==1=$

13. Fibula

14. Humerus . . . . mit $3==1=$

15. Scapnla $\}$. . . mit je $2==0,6=$

Für die Skeletabschnitte stellt sich folgende Reihenfolge heraus:

1. Untere Extremität 160 Fälle $=50,6$ Proc.

2. Kopf nnd Stamm $100=-31,5=$

3. Obere Extremität $57==17,9=$

Vergleichen wir mit diesen Zahlen diejenigen Billroth's und Müch's, so finden sich folgende Verhältnisse. Billroth und Menzel beobachteten folgende Reihenfolge:

1. Wirbelsäule.

9. Handknochen.

2. Kniegelenk.

3. Schädel und Gesicht.

10. Tibia und Fibula.

4. Hüftgelenk.

11. Femur.

5. Sternum und Rippen.

6. Fussknochen.

7. Ellenbogengelenk.

12. Schultergelenk.

13. Hnmerus.

8. Beckenknochen.

14. Ulna.

15. Radius.

16. Scapula.

Münch dagegen fand:

a) Caries.

1. Rumpf . . . . . . $=33,6$ Proc.

2. Untere Extremität . . $=33$

3. Obere $\quad . \quad \cdot=22,3=$

4. Kopfknochen . . . . $=8,3=$

5. Multiple Caries . . . $=2,2=$

b) Tumor albus.

1. Untere Extremität . . $=74,3$ Proc.

2. Obere $=. .=25,7=$ 
Wie man sieht, nehmen die meisten der angegebenen Knochenregionen in Billroth's und unserer Reihe dieselbe Stelle ein, und wir werden nicht fehl gehen, aus dieser Uebereinstimmung einen Rückschluss auf die Richtigkeit der beiden, auf so verschiedene Weise gewonnenen Angaben zu machen. Als besonders abweichendes Ergebnis wären nur die Kopfknochen zu nennen, die bei uns erst in achter Reihe kommen, während Billroth's Statistik sie schon in dritter Linie auffuhrt. Es erklärt sich dies wohl ziemlich leicht aus den fehlenden Kindern und Heilungen in Billroth's Fällen. Die zwischen Wirbelsäule und Kopfknochen bei uns vorkommenden Knochengruppen, die Fuss-, Hüft-, Hand- und Ellenbogenknochen, pflegen bei Kindern besonders gern tuberculös zu erkranken; ich erinnere nur an die Caries der Fusswurzel, Coxitis, Spina ventosa und Caries olecrani. Gerade diese Affectionen (mit bedingter Ausnahme vielleicht der Coxitis) fuhren selten zum Tode, so dass wohl anzunehmen ist, dass in der Häufigkeit des Vorkommens überhaupt die Kopfknochen eher an die achte, als an die dritte Stelle zu setzen sind. Dass die Tuberculose der Fussknochen bei uns in zweiter, bei Billroth erst in sechster Linie aufgefuhrt ist, erklärt sich wohl ebenfalls aus den vielen Heilungen wir hatten deren 91 Proc. - bei diesen Affectionen.

Die Münch'sche Statistik mit einer Procentzabl von Rumpf und Kopf zusammen $=41,9$ gegen 33 der unteren Extremität, weicht nur scheinbar von der unserigen mit dem Verhältniss von $31 \frac{112}{2}$ :50,6 Proc. $a b$, da $M \ddot{t} n c h$ den Tumor albus der Gelenke besonders rubricirt hat; zählt man diese Fälle zu den obigen hinzu, so stellt sich das umgekehrte Verhältnis zwischen Rumpf und Kopf und unterer Extremität wieder her.

Eine besondere Aufmerksamkeit verdienen die tuberculösen Spondylitiden, die auch von Billroth und Menzel eingehender gewürdigt worden sind. Die nachfolgende Curve 2 (S. 442) zeigt zunächst ihr Vorkommen an den einzelnen Wirbeln; letztere sind durch die Ordinaten bezeichnet, während die Abscissen hier die procentische Berechnung der Fälle repräsentiren. Die obere Curve bezieht sich auf alle zur Beobachtung gekommenen Spondylitiden, während die untere punctirte nur die zur Autopsie gelangten umfasst.

Während bei dieser die Akme auf die letzten Lendenwirbel fällt, liegt sie bei jener in den Brustwirbeln. In der von Billroth entworfenen, auf 702 Fällen basirenden Curve steht der zweite Halswirbel obenan, dann folgen die mittleren Brustwirbel und zuletzt die 
letzten Lendenwirbel. Es ist nicht ersichtlich, warum in Wien der zweite Halswirbel so häufig erkrankt gefunden wurde; bei uns ist dies sicher nicht der Fall; auf 10 dorsale kommt kaum 1 cervicale Spondylitis. Im Uebrigen stimmen Billroth's und unsere obere Curve überein. Der Grund für das häufigere Erkranken der Brustwirbel ist vielleicht, wie Billroth (1. e. S. 356) meint, auf ihre grössere Zahl gegenüber den Hals- und Lendenwirbeln zurückzufübren.

Curve 2.

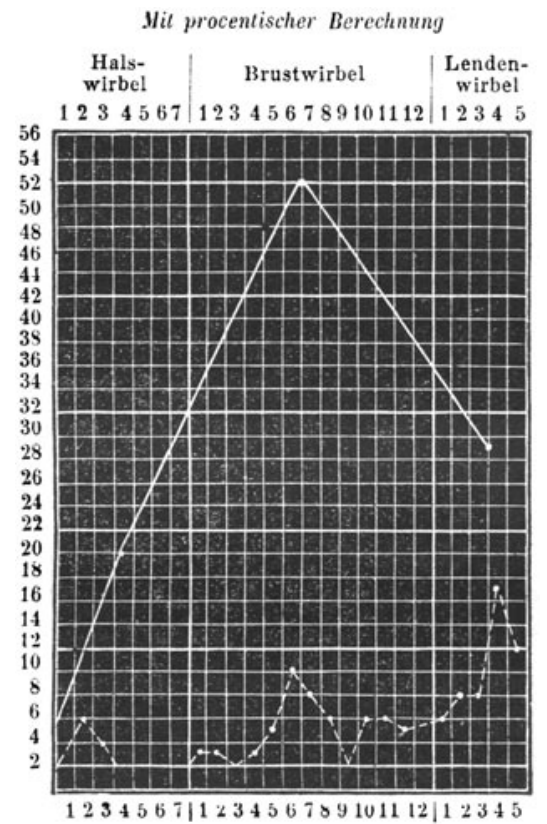

Vergleichende graphisehe Darstellung der Häufigkeit tuberculöser Spondylitiden bei 22 Fällen gestorbener Sp. mit 55 erkrankten Wirbeln und 82 Fällen von Sp. überhaupt, beide procentisch umgerechnet. Die Gestorbenen sind punctirt, die anderen ausgezogen gezeichnet.

Nächst der Wirbeltuberculose stehen die tuberculösen Erkrankungen der Fussknochen obenan. M ü nch (1. c. S. 352) fand unter 265 Cariesfällen die Fussknochen $81 \mathrm{mal}=30,5$ Proc. erkrankt, also noch etwas häufiger, während Billroth von 1996 nur 150 Fälle angibt, also 7,5 Proc. Die Grïnde für diese auffallende Abweichung sind bereits oben erwähnt. Wir duirfen wohl ziemlich sicher, auf Grund der Münch'schen und unserigen Statistik die Fussknochen in zweiter Linie verzeichnen.

Die übrigen Ergebnisse der Curve sprechen für sich selbst und bedïrfen keiner weiteren Erläuterung. 


\section{Geschlecht und Alter.}

Unter unseren 317 Fällen befanden sich

$$
\begin{aligned}
& 179=561 / 2 \text { Proc. männlichen und } \\
& 138=431 / 2=\text { weiblichen Geschlechts. }
\end{aligned}
$$

Dies Ergebnis, das ungefähr einem Verhältnis von $3: 2,3$ entspricht, weicht von dem von Billroth und Menzel gefundenen, wo auf 1295 Männer nur 811 Weiber kommen, also ein Verhältnis von $13: 8$, recht erheblich ab. Die Differenz erklärt sich aus folgendem Grunde.

Männer mit leichteren tuberculösen Knochenerkrankungen, die in der Regel ausheilen, gehen nicht so leicht in's Spital wie Weiber; man wird immer unter den geringgradig Erkrankten mehr Weiber als Männer finden. Da für die Kinder, die in der Bill roth'schen Statistik fehlen, das Geschlecht keinen Unterschied zu machen pflegt, so wird in einer Statistik, die Geheilte und Gestorbene umfasst, eine grössere Anzahl Weiber figuriren müssen, als in einer ausschliesslichen Sectionsstatistik.

Von den einzelnen Knochengruppen sei nur das Kniegelenk hervorgehoben, das bei fast doppelt so viel Männern als Weibern tuberculös afficirt gefunden wurde. Ob hier mehr Zufall vorliegt, ob die Traumen, denen dies Gelenk gerade bei Männern so vielfach ausgesetzt ist, die Schuld daran tragen, lässt sich wohl kaum ergrüunden.

Für das Alter, in dem sich unsere Kranken befanden, ergibt sich, dass fast die Hälfte aller Fälle, nämlich $136=43$ Proc., auf das erste Lebensjahrzehnt entfällt, während die ïbrigen Jahrzehnte mit

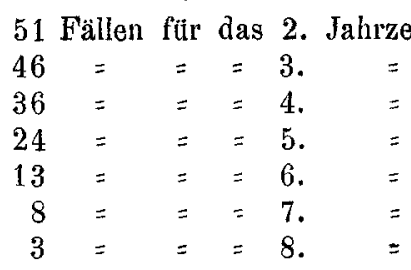

stetig abnehmen. Der hohe Procentsatz der ersten 10 Lebensjahre erklärt sich nicht etwa allein aus der stärkeren Mortalität dieser Periode, speciell an Knochentuberculose, sondern direct aus dem häufigeren Auftreten der letzteren in diesem Alter. Während die Tuberculose innerer Organe am häufigsten das 3. Jahrzehnt, also vom 20. bis 30. Jahre, zu befallen pflegt, tritt die primäre Knochentuberculose in diesem Alter schon mehr zuritck. Die Spondylitis allein figurirt bei uns mit 51 Fällen $=62$ Proc. in der ersten Lebensdekade. Um $\mathrm{zu}$ ermitteln, wie sich in dieser für die Knochentaberculose wichtigsten 
Altersperiode die einzelnen Jahre verhalten, wurde noch die folgende Curve 3 construirt, die zu interessanten Ergebnissen geführt hat.

Curve 3.

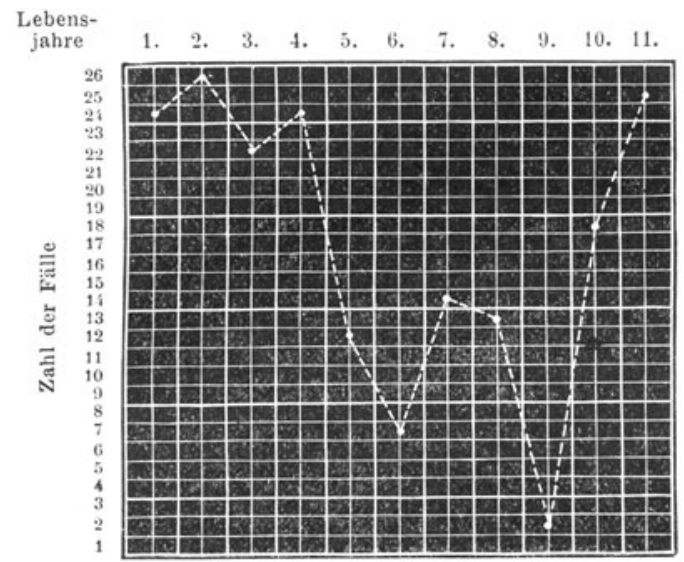

Graphische Darstellung der Hänfigkeit der Knochentubereulose während der ersten 10 Lebensjahre bei 136 Fällen. Die absolute Zahl der Beobachtungen ist eingezeichnet.

Dieselbe zeigt in den Ordinaten die ersten 10 Lebensjahre, und zwar so, dass unter "1. Jahr" die ersten 12 Lebensmonate, unter 2. die zweiten u. s. f. verstanden sind. Die Abscissen stellen wieder die absolute Zahl der Beobachtungen dar. Man erkennt sofort, dass das 3. Lebensjahr, also dasjenige Alter, wo man im gewöhnlichen Sprachgebrauch die Kinder ${ }_{n} \mathrm{zw}$ ei "Jahre alt nennt, bei Weitem das grösste Contingent an Erkrankungen stellt; ihm folgt

\begin{tabular}{c|r} 
das 6. Jahr & 9. Jahr \\
dann das $7 .=$ & $8 .=$ \\
$4 .=$ & $11 .=$ \\
$5 .=$ & $1 .=$ \\
2. & und $10 .=$
\end{tabular}

Auf die letzten drei Zahlen mag man kein besonderes Gewicht legen; sie sind zu klein, um den Zufall sicher ausschliessen zu können. Aber die hohe Ziffer für das 3. Lebensjahr ist doch recht wichtig und interessant. Nach C. Hueter ${ }^{1}$ ), Schtuller ${ }^{2}$ ) u. A. kommen tuberculöse Gelenkprocesse angeboren gar nicht, im 1. und 2. Lebensjahre aber nur äusserst selten vor. Schüller sah unter 232 Fällen "genau constatirter" Gelenktuberculose nur eine einzige Spondylitis vor dem 3. Lebensjahre. Die im 1. und 2. Lebensjahre "den tuberculösen äusserlich ähnlichen" Gelenkprocesse sind

1) Klinik der Gelenkkrankheiten. 2. Aufl. I. Bd. 1876. \$128.

2) 1. c. S. 171 . 
nach Schttller auf hereditäre Lues zu beziehen. Letzteres ist, wenigstens in so exclusiver Form, nicht richtig. Unter unseren 11 Fällen von Knochen- und Gelenktuberculose, die vor dem 3. Lebensjahre auftraten, finden sich fün $\mathbf{f}$ Gelenkprocesse unzweifelhaft tuberculöser Natur, soweit dies wenigstens durch Messer und Mikroskop nachgewiesen werden kann. Dass gerade das 3. Lebensjahr dann die grösste Anzahl von Erkrankungen anfweist, spricht ebenfalls für ein frühes Auftreten der Tuberculose, da die Kinder oft erst nach Monaten zum Arzte, oder wenigstens ins Krankenhaus gebracht werden, wo sich dann der Anfang des Processes gar nicht mehr genau feststellen lässt. Unsere juingste Patientin war 8 Wochen alt: Clara Mellmann, vom 1. bis 4. Juli 1880 im Krankenhause und dann gestorben. Die Section ergab eine ausgesprochene tuberculöse Caries des 4. Lendenwirbels, dessen Körper völlig in käsiges Gewebe umgewandelt war. Hier kann beinahe eine congenitale Knochentubereulose angenommen werden, da in der kurzen Zeit von 8 Wochen eine ausgesprochene Caries sich kaum zu entwickeln vermag. Leider steht der Fall in unserer Reihe vereinzelt da, so dass er für die Frage nach der angeborenen Tuberculose nicht weiter verwerthet werden konnte.

\section{Ueber die Heilungen der Knochentuberculose.}

Der Ausgang der Knochen- und Gelenktuberculose ist entweder in Heilung, chronisches Siechthum oder Tod. Die Heilung kann spontan erfolgen oder nach Zerstörung resp. Entfernung der Krankheitsherde. Tritt keine Spontanheilung ein und 'der tubereulöse Herd wird nicht aus dem Körper eliminirt, so erfolgt durch innere Metastasen oder amyloide Degeneration nach kürzerer oder längerer Zeit der letale Ausgang.

Die von uns als "ungeheilt" aufgeführten 12 Fälle sind so zu erklären, dass die betreffenden Patienten vor dem Abschluss des guten oder schlechten Krankheitsverlaufes das Spital verliessen und auf diese Weise unserem Gesichtskreis entschwanden; die übrigen sind entweder als - zur Zeit ihres Austritts - Geheilte entlassen, oder im Krankenhause gestorben und secirt.

Wir wollen in diesem Abschnitt einen Blick auf die geheilten Fälle werfen und zugleich auf die Art und Weise, wie dieselben zu Stande gekommen, etwas näher eingehen.

Im Ganzen hatten wir 245 Heilungen, also 77 Proc. aller Fälle, 
zu verzeichnen, was gewiss ein günstiges Resultat genannt zu werden verdient. Von diesen 245 Fällen wurden geheilt

1. ohne operativen Eingriff . . . . . $82=32$ Proc. aller Fälle

2. durch Gelenkauswaschung . . . $18=7==$

$3 .=$ Incision und Evidement. . . $69=27==$

$4 .=$ Resection . . . . . . $56=22==$

$5 . \quad=$ Amputation oder Exarticulation . $16=6==$

6. ungeheilt blieben... . . . . $12=4==$

Von den sub 3 bis 5 genannten, operativ behandelten Kranken heilten

a) nach einem operativen Eingriff . . $108=42$ Proc.

b) nach mehreren operativen Eingriffen . $35=14=$

Die Frage nach der besten therapeutischen Methode der Knochentuberculose ist durchaus noch nicht abgeschlossen, und mannigfache Widersprüche begegnen uns in den Angaben der zuverlässigsten Beobachter. In einem Punkte sind so ziemlich alle Autoren einig, nämlich in dem Satz: "Alles Kranke muss grïndlichst entfernt werden," aber über das Wie und Wann dieser Entfernung herrschen grosse Differenzen.

Dass Spontanheilung bei ausgesprochener Knochentuberculose vorkommen kann, ist zwar nicht allgemein anerkannt, aber fuir uns ausser allem Zweifel. Gegen die Möglichkeit lässt sich a priori nichts einwenden: wenn ein Lungentuberkel verkreiden oder verkalken, oder eine Caverne veröden und vernarben kann, warum soll nicht auch ein beschränkter tuberculöser Knochenherd ausheilen können? Sichere Beobachtungen über solche Spontanheilungen citirt König ${ }^{1}$ ), wo bei Kindern (ein Tumor albus genu, zwei Coxitiden) obne Resection Heilung eintrat. Boegehold ${ }^{2}$ ) hält die von Scriba ${ }^{3}$ ) und Rinne ${ }^{4}$ ) veröffentlichten Fälle für solche Spontanheilungen und erinnert an die Congestionsabscesse der Spondylitis, die ebenfalls tuberculöser Natur sind und ausheilen können. Er glaubt eine gutund bösartige Form der localen Tuberculose danach annehmen zu können, womit jedoch für die wissenschaftliche Erklärung nichts gewonnen wird. Als bester Bewéis für die Möglichkeit dieses Ausganges ist die tuberculöse Spondylitis selbst zu nennen, bei der wir in fast 50 Proc. durch die tibliche Extensionsbehandlung Heilung erzielten. Von den übrigen Knochengruppen sind noch Hüft- und Knie-

1) Verhandlungen der deutschen Gesellschaft für Chirurgie. X. 93.

2) 1. c. S. 732 .

3) Berliner klin. Wochenschrift. 1877. Nr. 32:

4) Centralblatt für Chirurgie. 1877. 
gelenk und die Fussknochen zu nennen, bei denen wir 12-, resp. 8 mal anf diese Weise Heilung eintreten sahen.

Als mildester operativer Eingriff wäre zunächst die parenchymatöse Injection, speciell von Carbolsäure, zu nennen, die besonders von C. Hu eter und seinen Schülern in die Chirurgie eingeführt worden ist. Sie versprach anfangs mehr, als sie später geleistet hat. König's ${ }^{1}$ ) Prophezeinng, die medicamentöse Injection sei für die locale Tuberculose die Therapie der Zukunft, hat sich nicht bewährt. Immerhin sind damit nennenswerthe Erfolge erreicht worden, und speciell bei manchen leichten Tumor albus-Formen und beginnender Tuberculose der Hand- und Fusswurzelknochen haben auch wir mehrere Heilungen damit erzielt.

Von der Incision und Drainage glaubt Boegehold ${ }^{2}$ ), der ein eifriger Gegner der Ignipunctur, Evidements und Resectionen ist, bei Gelenktuberculose Erfolge gesehen zu haben, gesteht aber selbst zu, dass das eigentliche Gebiet der Gelenkdrainage bei anderen Gelenkkrankheiten zu suchen sei.

Ist es einmal zur Eiterung gekommen, so treten die energischeren Eingriffe in ihre Rechte. Die e infache Incision genügt höchstens bei Congestionsabscessen, wo der primäre Herd nicht zu erreichen ist; bei Spondylitis sind damit manche Erfolge erzielt, obgleich $\mathrm{König}{ }^{3}$ ) auch hier angefangen hat, dem Ausgangsherd möglichst direct $z u$ Leibe zu gehen.

Bei kleineren, leicht zugänglichen Herden genügt das Evidement mit Löffel oder Glüheisen; bei grösseren bedarf es der Ausmeisselung oder Resection. Eine vielfach ventilirte Frage ist es, ob man möglichst frïh reseciren soll oder erst warten, bis der Herd in's Gelenk oder nách aussen durchgebrochen ist. König ${ }^{4}$ ) war der Erste, der auf Grund eines grösseren Resectionsmateriales die schlechten Erfolge der Frühresection nachwies; er formulirte ihre Indication später ${ }^{5}$ ) dahin, dass sie nur da angezeigt sei, wo es sich blos um Erhaltung der Gelenkfunction handele, nicht aber wenn die Allgemeininfection verhindert werden soll. Danach wäre sie bei wirklicher Tuberculose der Gelenke oder Knochen contraindicirt. In seiner letzten Arbeit (25) kommt er wieder auf die Nothwendigkeit einer früheren Operation zurück, die ohne Rücksicht auf Gelenkeröffnnng vorzunehmen sei, auch da, wo es noch nicht zur Eiterung

$\begin{array}{ll}\text { 1) Diese Zeitschrift. Bd. XI. } 570 . & \text { 2) I. c. S. } 727 .\end{array}$

3) Berliner klin. Wochenschrift. 1880. Nr. 7.

4) Langenbeck's Archiv. XXV. 580.

5) König, Verhandlungen der deutschen Gesellschaft für Chirurgie. X. 96. 
gekommen. Er steht jetzt unter dem Einfluss des Jodoforms und hofft, mit demselben auch bei Resectionen weit bessere Resultate erzielen zu können, als bisher. Lossen (26), im Allgemeinen ein eifriger Anhänger der Frühresection, verwirft sie nur für den Fuss; für Ellenbogen- und Schultergelenk sei sie in jedem Alter zulässig, während Hand-, Knie- und Hüftgelenk im jugendlichen Alter gute Resultate abgeben. Herr Dr. Scbede ist in den letaten Jahren ein entschiedener Anhänger der Frühresection geworden, seitdem er von den partiellen Resectionen, zumal bei Kindern, ausgezeichnete Resultate gesehen hat. Es gelingt oft, nur durch Aufmeisselung des betreffenden Condylus den tuberculösen Herd zu entfernen und eine Heilung herbeizuführen, ohne iiberhaupt das Gelenk eröffuen zu müssen.

Neben der localen Herdbeschaffenheit ist jedoch der Allgemeinzustand und das Alter wesentlich mit zu berïcksichtigen. Bei gesunden inneren Organen wird man bei jugendlichen Individuen durch gründliche Entfernung des tuberculösen Localherdes, sei dies nun durch Evidement, Ausmeisselung oder Resection, für die definitive Heilung günstige Chancen haben; für die spätere Function des betreffenden Gliedes wird natürlich ceteris paribus das conservativere Verfahren vorzuziehen sein. Sind aber Lungen oder sonstige Organe (worunter ich besonders auch die benachbarten Lymphdriasen nennen möchte) bereits tuberculös erkrankt, so wird man höchstens im kindlichen Alter von einer Resection noch zuweilen Erfolge sehen. Bei Erwachsenen tritt hier die Amputation als diejenige Methode, welche am gründlichsten alles Kranke entfernt, in den Vordergrund. Dass allgemeine Scrophulose, Tuberculose oder Amyloid im Allgemeinen keine Contraindication für die Amputation abgeben, ist auch von König (21) und Schede (33) urgirt worden. Besonders häufige Recidive sah Münch (l. e.) nach partiellen Fussamputationen wegen tuberculöser Caries. Speciell die Lisfranc'sche, Chopartsche und Pirog off'sche Amputation hatten locale Recidive, multiple Caries oder allgemeine Tuberculose im Gefolge, während die Symesche Operation zwar längere Heilungen erzielte, aber functionell ungünstige Resultate lieferte. Auch Schede (34, S. 85) fand als Resultat einer vergleichenden Zusammenstellung der functionellen Misserfolge bei den genannten Operationen (nach Abzug der Todesfälle)

$$
\begin{gathered}
\text { fuir Pirogoff } 7,9 \text { Proc. } \\
=\text { Chopart } 9,5= \\
=\text { Syme } 10=
\end{gathered}
$$

wobei jedoch nur diejenigen Fälle berücksichtigt sind, bei welchen 
unvollständige Heilungen (Fisteln), Nachamputationen u. s. w. unmittelbar nach der Operation beobachtet wurden. Rechnet man ferner die wegen anderen Affectionen, als tuberculöser Caries, Amputirten von der Ziffer der Heilungen ab, so werden wir auch hier eine viel höhere Procentzahl der Misserfolge zu verzeichnen haben. C. Hueter (13) glaubte, die "scrophulösen " Recidive nach den Resectionen entständen aus zwei Urachen: theils aus zurïckgebliebenen tuberculösen Massen, theils durch die allgemeine scrophulöse Dyskrasie. Gegen erstere empfahl er Einschieben von Protective oder Jodoformstäbchen in die Fisteln, häufig wiederholte Ausschabungen oder Ausbrennen; gegen letztere eine roborirende Allgemeinbehandlung. Auch Schüller $(36$, S. 224) betont die Wichtigkeit der letzteren; ausser den Mitteln, die den Gesammtkörper kräftigen, soll man die causal wirkenden nicht vergessen, wozu nach seinen Versuchen das Natriumbenzoat in erster Linie zu rechnen sei.

Ueberblickt man die Ergebnisse, welche sich aus den Angaben der genannten Autoren für die Heilung der Knochentuberculose gewinnen lassen, so gelangt man, meiner Meinung nach, zu folgenden Sätzen:

Die Knochen- resp. Gelenktuberculose ist entweder auf den Ausgangsherd local beschränkt, oder sie ist in mehreren Knochen gleichzeitig localisirt, oder es besteht gleichzeitig eine Tuberculose innerer Organe. In den ersten beiden Fällen kann bei zweckmässiger expectativer Behandlung, so lange es noch nicht zur Bildung von Eiter oder käsigen Sequestern gekommen ist (vgl. König [25] S. 5) eine Spontanheilung erfolgen; dieselbe kann durch medicamentöse Injectionen noch befördert werden. Bleibt die Heilung aus, tritt Eiterung u. s. w. ein, so muss, soweit irgend möglich, der tuberculöse Herd anfgesucht und entfernt werden; ob hierbei das Evidement, die Resection, Amputation oder Exarticulation angezeigt sei, entscheidet das Alter des Patienten, die Lage des Herdes und seine Beschaffenheit. Für die Nachbehandlung wird in einzelnen Fällen Jodoform, Sublimatsand ${ }^{1}$ ) oder Wismuth ${ }^{2}$ ) wesentliche Unterstützung für die Heilung gewähren können. Findet sich aber bereits eine ausgesprochene tuberculöse Erkrankung innerer Organe (speciell der Lungen) oder der Lymphdrüisen, so ist bei leidlichem Allgemeinzustand des Patienten vielleicht noch von der Amputation des afficirten Gliedes eine Heilung zu erwarten. In solchen Fällen ist eine besonders sorg-

1) Nach $\mathrm{K}$ ümme11, Verhandl. der d. Gesellschaft für Chir. XI. Congress.

2) Nach Kocher, Centralblatt für Chirurgie. 1882. Nr. 15. S. 235. 
fältige allgemeine und - soweit dieselbe nach unseren heutigen Erfahrungen möglich ist - antituberculöse Behandlung am Platze.

\section{Ueber die Todesarten bei Knochentuberculose.}

Der Tod kann bei Knochentuberculose aus den verschiedensten Ursachen erfolgen. Die Kranken gehen entweder durch die lange Eiterung an Marasmus und Erschöpfung zu Grunde, oder es bilden sich diffuse chronische Erkrankungen der grossen Unterleibsdrüsen, als Fettleber, Morbus Brightii und ähnliche heraus, oder es kommt zu allgemeinem Amyloid aller drüsigen Apparate; oder eine intercurrente tuberculöse Meningitis macht den Leiden ein Ende; oder endlich es entwickelt sich eine mehr oder weniger ausgedehnte innere Tuberculose mit käsigen Herden, Ulcerationen und Tuberkelablagerungen.

Für die Häufigkeit des Vorkommens dieser einzelnen Eventualitäten ist Billroth-Menzel's (l. c.) Statistik besonders verwendbar, auf die hier verwiesen sei. Von den Hauptresultaten derselben sei nur erwähnt, dass in 22 Proc. der Fälle keine inneren Befunde nachzuweisen waren; die Unterleibsdrüsen fanden sich bei 26,1 Proc. chronisch diffus erkrankt; käsige Herde, Ulcerationen, Cavernen und innere Tuberkel kamen dagegen in 54,2 Proc. der Fälle vor. Diese hohe Ziffer wird von Billroth (l. c. S. 365) daraus erklärt, dass diese letzteren Befunde in seiner Statistik nicht etwa nur Folgen der Knochencaries waren, sondern dass oft beide Zustände, Caries und Tuberkel, aus einer und derselben Grundursache, „etwa der Scrophulose " entstanden seien.

Für uns hatte die Frage nach der Häufigkeit der allgemeinen 1) Tuberculose und allenfalls noch des Amyloids im Gefolge der Knochentuberculose ein besonderes Interesse, weil darüber, wie gleich zu erwähnen, auch von anderen Autoren Angaben existiren, und wir haben daraufhin unser Material besonders durchforscht. Unter nnseren 60 Gestorbenen und Secirten fand sich bei 29, also fast in der Hälfte, allgemeine inmere Tuberculose; Amyloid mit oder ohne Tuberkel dagegen nur bei $20=33$ Proc. Dem Procentsatz nach stellte die Tuberculose der Schädelknochen, Wirbelsäule, des Ellenbogen- und Kniegelenks das grösste Contingent, während Amyloid bei der Coxitis tuberculosa am häufigsten auftrat. Ein richtiges $\mathrm{Ur}^{\mathrm{r}}$ -

1) Darunter ist immer nur die $\operatorname{chr} \mathbf{r}$ is che Tuberculose, nicht die acute miliare zu verstehen. 
theil gewinnt man jedoch erst, wenn man diese Zahlen in ihrem Verhältnis zur Gesammtziffer vergleicht, wobei für die allgemeine Tuberculose nur 9 Proc., für das Amyloid nur 6 Proc. herauskommen.

Dies sind recht günstige Resultate und übertreffen die friiher von Billroth, König und Münch gefundenen Zahlen nicht unerheblich.

Nach Billroth $\left.{ }^{1}\right)$ starben an Tuberculose, gemäss einer 16jährigen Statistik, von Gelenktuberenlösen mit oder ohne Operation 27 Proc. - König ${ }^{2}$ ) hatte unter 117 Resecirten $18==16,3$ Proc. Todte mit allgemeiner Tuberculose; zu diesen kommen jedoch noch 9 (damals) Lebende hinzu, welche Symptome allgemeiner, unheilbarer Tuberculose darboten, so dass eine Gesammtzahl von 23 Proc. (nicht 21,5 Proc., wie es bei $\mathrm{K} \ddot{\mathrm{n}} \mathrm{ig}$ irrthümlicher Weise heisst) herauskommt.

Nicht viel anders lauten die Resultate $M \ddot{u} \mathrm{ch}^{\prime} \mathrm{s}^{3}$ ) bei Caries der Fussknochen. Eine Zusammenstellung von 50 theils im Spital, theils später ausserbalb erfolgten Todesfällen ergab Münch, dass die Todesursachen sich in vier Gruppen eintheilen liessen. Es starben nämlich

1. an intercurrenter (acuter) Miliartuberculose . . $5=10$ Proc.

2. an Lungenphtbise . . . . . . . . . . $19=38=$

3. an chronischer Erschöpfung und Folgen . . , $23=46=$

4. an zufälligen Krankheiten (Tetanus, Ileus, Typhus) $3=6=$

Auch seine sonstigen Ergebnisse verdienen noch eine besondere Erwähnung. Von 81 Fällen mit Fusscaries starben $17=15$ Proc., während bei uns nur 9 Proc. erlagen. Jene 17 Todesfälle vertheilen sich folgendermaassen:

1. Von 27 expectativ Behandelten starben $4=15$ Proc.

2 . $=13$ mit Evidement $=\quad=2=15=$

$3 .=45$ Resecirten. . . . . $=5=11=$

4. $=44$ Amputirten . . . $=6=14=$

Unter den als "geheilt" Entlassenen liess sich später allgemeine Tuberculose nachweisen:

1. Von 15 expectativ oder mit Evidement Behandelten bei $1=7$ Proc.

$2 .=20$ Resecirten . . . . . . . . . $=4=20=$

3. $=33$ Amputirten . . . . . . . . . $=8=24=$

Man ersieht aus diesen Zahlen zunächst, dass die Mortalität Mü nch's bei den verschiedenen Behandlungsmethoden nicht wesentlich differirt und im Mittel circa 14 Proc. beträgt, während wir für

1) Bill roth, cit. bei König, Langenbeck's Archiv. XXV. 1880.

2) 1. c. S. $580 . \quad$ 3) l. c. S. 383 . 
alle Fälle von Knochentuberculose zusammen die nicht viel böhere Mortalitätsziffer von 19 Proc. gefunden haben.

Wesentlich anders gestalten sich aber die späteren Folgen bei Mỉnch's Fällen; nur die leichteren, spontan oder durch Evidement geheilten Kranken blieben gesund; von den uibrigen wurden zusammen 44 Proc., also fast die Hälfte aller Fälle, tuberculös, eine Zahl, die sogar diejenige König's fast um das Doppelte übertrifft.

Wir können und dürfen uns, nach diesen gewiss zuverlässigen Angaben, der Thatsache nicht verschliessen, dass alle diejenigen Kranken mit Knochentuberculose, die der Resection oder gar Amputation verfallen sind, bei denen also der primäre Herd nicht mehr durch Spontanheilung oder Evidement ausheilen kann, im hohen Grade der Gefahr ausgesetzt sind, an allgemeiner Tuberculose zu Grunde $\mathrm{zu}$ gehen, und wir mijssen, trotz vielfach gegentheiliger Angaben und trotzdem unsere eigene Statistik eine so günstige Ziffer für die - unmittelbare - Heilung ergeben hat, an dieser schlechten Prognose festhalten, sobald die Knochenaffection in das erwähnte Stadium getreten ist.

\section{Ueber das Verhältnis der Knochentuberculose zur allgemeinen Tuberculoso.}

Die Frage nach dem Verhältnis der chronischen Knoehentuberculose zur allgemeinen Tuberculose ist heute, wie wir bereits eingangs erwähnten, im Grossen und Ganzen als gelöst zu betrachten. Trotzdem tauchen immer noch wieder Zweifler auf, welche von einer Identität zwischen den einzelnen Tuberculoseformen nichts wissen wollen und zwischen "localer" und "allgemeiner" Tuberculose einerseits, und "käsiger" und "tuberculöser" Entzündung andererseits eine Grenze ziehen. Man hat die Vertreter der letzteren Ansicht passend als $\mathrm{Dualisten}$ in der Tuberculosefrage bezeichnet und ihnen die Unitarier gegenüber gestellt. Ich glaube, dass es für Jeden, der heute über Knochentuberculose schreibt, fast unerlässlich ist, seinen Standpunkt in der beregten Frage zu fixiren. Ich halte, wie ich gleich bemerken will, den Unitätsstandpunkt für den allein richtigen und will versuchen, dies mit wenigen Worten zu begründen.

Zunächst muss ich vorausschicken, dass die $\mathrm{K} 0 \mathrm{ch}$ 'sche Entdeckung des Tuberkelbacillus, so wichtig dieselbe für die ätiologische Auffassung der Tuberculose ist, für unseren Standpunkt ausser Acht gelassen werden kann. Allerdings hat ja Koch (l. e. S. 223) in vereinzelten Riesenzellen fungöser Gelenke Bacillen gesehen, während im hiesigen Krankenhause (und, wie ich höre, ebenso im Strass- 
burger und Wiener pathologischen Institut) bei einer grossen Reihe frisch exstirpirter tuberculöser Gelenkkapseln nicht ein einziges Mal dieselben gefunden worden sind. Aber was beweist dies? Auch im Sputum echt Tuberculöser hat man Bacillen vermisst und bereits angefangen, von "bacillärer " und "nichtbacillärer" Phthise zu sprechen. Solche Aufstellungen verwirren nur die Vorstellungen von der Krankheit und erschweren das Verständnis der einzelnen Formen. Der Begriff "Tuberculose", wie er hente gebraucht wird, ist nun einmal keine ätiologische, sondern eine klinisch-anatomische Einheit, und wir können Schottelius (47, S. 154) nur Recht geben, wenn er, statt zu fragen: "Ist die Tuberculose eine Infectionskrankheit," die Fragestellung umdreht und fragt: "Gibt es eine infectiöse Tuberculose?" Die letztere Frage ist auf Grund der Koch'schen Untersuchungen sicher zu bejahen, aber ebenso sicher muss man sagen, wenn in einer Form der Tuberculose die Bacillen nicht gefunden werden und es auch nicht gelingt, das Cohnheim'sche Postulat: "Mit dem Gewebsmaterial im Versuchsthier Tuberculose zu erzeugen, " zu erfüllen, so gehört die betreffende Krankheit dennoch zur Tuberculose, wenn sie deren klinische und anatomische Bilder darbietet.

Von diesem Standpunkte aus kann es nicht schwer fallen, die Stellung der Knochen- und Gelenktuberculose richtig zu fixiren, wenn wir einen kurzen Blick auf ihre Geschichte werfen. - - Das Vorkommen von Tuberkeln in der Synovialis beschrieb zuerst Rokitansky (32); seine Angaben bestätigten später Virchow (40), Crocq (8) und Volkmann (42); doch galten diese Tuberkel nur als Theilerscheinung der acuten Tuberculose. Im selben Sinne fanden und beschrieben das Knötchen Suren ${ }^{1}$ ), Nélato $n^{2}$ ), Stanley ${ }^{3}$ ), Lebert ${ }^{4}$ ) und Virchow (41) im Knochen und gaben als seinen vornehmlichen Sitz das Knochenmark an. Köster (17) ging einen Schritt weiter und zeigte, dass fast in jedem Falle von fungöser Gelenkentzündung: Riesenzellen und Tuberkel sich nachweisen lassen. Seine Angaben wurden von König (21) und Volkmann (43) in der Folge bestätigt. Aber es traten Zweifel an der klinischen Uebereinstimmung mit den gewöhnlichen Tuberkeln auf. Friedländer (10) rersuchte zuerst, eine "locale" Taberculose aufzustellen, die sich durch ihren Verlauf von der "allgemeinen" unterscheiden soll. L ü cke (27)

1) De oss. tuberc. Diss. in Berolin. 1834.

2) Recherches sur l'aff. tub. des os. Paris 1837.

3) A treatise on diseases of the bone. London 1849.

4) Traité d'anat. pathol. T. II. Pl. CLXVI. 
machte eine Anzahl anderer, besonders acuter Infectionskrankheiten als Ursache für später auftretende fungöse Gelenkleiden geltend, und noch im Jahre 1881 versuchte Sonnenburg (38) aus Fällen von nicht ererbten tuberculösen Knochenherden den Satz herzuleiten: "Die meisten Fälle von Knochencaries sind zunächst entzündlicher Natur; sie entwickeln sich nicht auf dem Boden einer tubereulösen Constitution."

Wir dürfen heute diese Zweifel wohl als widerlegt ansehen. Die Lü cke'schen Einwände versuchte Schüller (36a) zu entkräften, indem er zeigte, dass keine der von Lü cke aufgeführten Krankheiten eine echte Knochen- oder Gelenktuberculose im Gefolge hat, während wir von P. Banggarten (1) erfahren haben, wie lange echt tuberculöse Herde latent im Organismus verweilen können, ehe sie in die klinische Erscheinung treten. Nach den sorgfältigen und zum Theil sehr mühseligen experimentellen und klinischen Arbeiten Schüller's (36), H. Hueter's (16) und König's (22, 25), besonders aber aus Volkmann's (43) musterhafter Darstellung dieses Gegenstandes können wir jetzt als feststehend annehmen, dass die früher als "Caries, chronische Ostitis, scrophulöse Knochenentzündung " u. s. w. bezeichneten Knochenleiden, ebenso wie der "Tumor albus, die fungöse, scrophulöse u. s. w. Gelenkentzuindung “ der Alten (mit einziger Ausnabme der nach Syphilis entstandenen) echt $t \mathfrak{u}$ berculöser Natur sind, die gewöhnlich von einem oder mehreren localen Herden ihren Ausgangspunkt nehmen und denselben deletären Einfluss auf ihre Umgebung und Nachbarschaft ausïben, wie der käsige Lungen- oder Hodentuberkel auf sein Mutterparenchym.

Es ertibrigt noch, zu erweisen, dass auch die Lungentuberculose - ebenso wie die primäre Tuberculose des Darms, des Urogenitalsystems, der Nebennieren, Lymphdrïsen, des Gehirns und der Haut - in ihrem Beginne immer als "locale" Formen der Tuberculose auftreten, um gezeigt zu baben, dass sie alle Erscheinungen eines und desselben Krankheitsprocesses sind, dessen Verschiedenbeiten durch die Natur des Bodens, auf dem er sich abspielt, bedingt sind. Ich kann dies mit wenigen Worten thun, da Heiberg (49) vor Kurzem erst diesen Beweis erbracht hat, und ich mich nur auf die von ihm zusammengestellten Thatsachen zu beziehen brauche.

Indem Heiberg an der Hand anatomischer Befunde die einzelnen oben genannten Formen primärer Tuberculose durchgeht, zeigt er zunächst, dass jede derselben aus einfachen, miliaren oder submiliaren Tuberkeln ihren Anfang nimmt. Man hat bisweilen bei 
plötzlichen Todesfällen aus äusseren Ursachen Gelegenheit, solche Anfangsstadien als "zufällige Befunde" in den Lungenspitzen oder anderen Prädilectionsstellen der Tuberkel zu constatiren. "Tuberkel fangen immer local an und inficiren nach und nach das Nachbargewebe" (1. c. S. 16). Auch die als "Peribronchitis" beschriebene Affection besteht aus cylindrisch oder röhrenförmig ausgebreiteten Tuberkeln, die auch durch ihre interalveoläre Lage ihre Zugehörigkeit zu denselben beweisen, während alle, katarrhalisch- und käsig-pnenmonischen Processe in traalveolär sich abspielen. In der Leber tritt derselbe Vorgang als tuberculöse Infiltration der Gallengangswandungen, in der Niere, Prostata, Hoden, Vas deferens, Ureteren und Tuben ebenfalls als peritubulöse Bildungen auf. Der Ausgang dieser Processe ist nun sehr verschieden. In seltenen Fällen kann auch der wirkliche Tuberkel durch fibröse Unwandlung zur Heilung kommen, wie dies von Grancher ${ }^{1}$ ) in einer Reihe von Arbeiten nachgewiesen worden ist. In anderen Fällen bleibt der tuberculöse Process auf das primär ergriffene Organ beschränkt und der Tod erfolgt im Marasmus oder durch die von der Tuberculose gesetzten Zerstörungen. So enden viele Formen unilateraler Phthise und manche Nierentuberculose. In einer dritten Gruppe von Fällen endlich generalisirt sich der Process früher oder später und macht durch eine tubereulöse Meningitis oder Miliartubereulose dem Leben ein Ende.

Jede Tuberculose ist also anfänglich eine locale Krankheit und es gibt keinen Grund, die Knochen- und Gelenktuberculose als eine specielle Form der Tuberculose anzusehen. Natïrlich gilt dieser Satz nur für die primären Formen, mögen dieselben nun im Knochen selbst oder der Synovialis ihren Anfang nehmen. Die als Theilerscheinung acuter Miliartuberculose auftretenden miliaren Knochentuberkel haben mit der von uns geschilderten Form nichts als den Namen gemein. Die primäre Knochen- und Gelenktuberculose aber gehört zur genuinen Tuberculose, wie die Urogenitaltuberculose und diejenige der Lymphdrüsen (Schüppel).

\section{Schlusssätze.}

Wir resumiren die Resultate der vorstehenden Mittheilungen in folgenden Sätzen:

1. Die Knochen- und Gelenktuberculose tritt an den einzelnen Knochengruppen des Körpers in folgender Häufigkeit auf:

1) Arch. de physiol. $1872-1878$. 


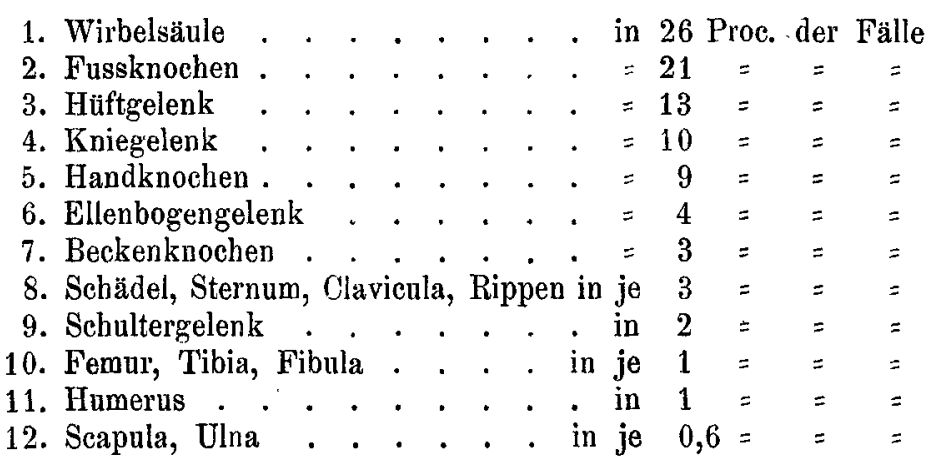

2. Die tuberculöse Spondylitis tritt am häufigsten an den Brustwirbeln, dann an den Lendenwirbeln und am seltensten an den Halswirbeln auf; die grösste Mortalität dagegen findet sich bei der Lumbalspondylitis, die geringste bei Cervicalspondylitis.

3. Die Zahl der männlichen Kranken bei Knochentuberculose steht zu den weiblichen im ungefähren Verhältnis von $3: 2,3$.

4. Die meisten Erkrankungen, nämlich 43 Proc., liefert das erste Jahrzehnt. Von diesen entfällt wieder die Mehrzahl auf das dritte Lebensjahr. Das fernere Vorkommen derselben nimmt mit den einzelnen Lebensdekaden in stetiger Progression ab.

5. Die Summe der Heilungen betrug bei 317 Fällen in toto 77 Proc., davon 32 Proc. ohne jeden operativen Eingriff, die Uebrigen nach einer oder mehreren Operationen.

6. Bei erwiesener Knochen- oder Gelenktuberculose ist die Frühoperation am Platze; sind die inneren Organe gesund und das Individuum jung, so kann dabei möglichst conservativ verfahren werden (Evidement, Arthrotomie, partielle oder totale Resection); im anderen Falle ist die Amputation angezeigt.

7. Von den Gestorbenen zeigte fast die Hälfte allgemeine Tuberculose, dagegen nur der dritte Theil Amyloid. Von 317 Kranken trat allgemeine Tuberculose bei 9 Proc., Amyloid bei 6 Proc. hinzu.

8. Die primäre Tuberculose der Knochen und Gelenke ist anatomisch und klinisch identisch mit der primären Tuberculose der inneren Organe, ebenso wie der Lymphdrüsentuberculose. Ob auch eine ätiologische Uebereinstimmung stattfindet, ist nicht ausgemacht, aber ohne Bedeutung für diejenige Auffassung der Tuberculose, welche in ihr keine ätiologische, sondern eine anatomisch-klinische Einheit erblickt. Für sie gibt es keine locale Tuberculose im Gegensatz zur allgemeinen, da jede primäre Form derselben als localer 
Process beginnt und häufig genug auch als solcher bis zu Ende verläuft.

Hamburg, März 1883.

\section{Literatur.}

1. Baumgarten, P., Ueber latente Tuberculose. Volkmann's klin. Vorträge. Nr. 218. 1882.

1a. Derselbe, Ueber die Wege der tuberculösen Infection. Zeitschrift für klin. Medicin. VI. $61 \mathrm{ff}$.

2. Bell, A treatise ete. " on white swellings of the joints. Edinburgh 1778.

3. Billroth, T., Allgemeine chirurg. Pathologie und Therapie. 7. Aufl. 1875.

4. Billroth und Menzel, Ueber die Häufigkeit der Caries u. s. w. Langenbeck's Archiv für klin. Chir. XII. S. 341. 1870.

5. Boegehold, Beiträge zur Pathologie und Therapie der Gelenkentzündungen. Ebenda: XXVII. S. 721. 1882.

6. Bonnet, Traité des maladies des articulations. Paris 1845.

7. Cohnheim, J., Die Tuberculose vom Standpunkte der Infectionslehre. 1880.

8. Crocq, Traité des tumeurs blanches. Bruxelles 1854.

9. Feurer, Anatomische Untersuchungen über Spondylitis. Virchow's Archiv. Bd. 82. S. 89. 1881.

9a. Frerichs, E., Beiträge zur Lehre von der Tuberculose. 1882.

10. Friedländer, C., Ueber locale Tuberculose. Volkmann's kl. Vortr. 64. 1873.

11. Geigel, Deutsch. Archiv für klin. Medic. XXV. S. 259. 1880. Die Macht der Analogie in der Lehre von der Infection.

12. Gross, System of surgery. 1862.

13. Hu eter, C., Verhandlungen der deutsch. Gesellschaft für Chir. X. 1881. S. 83.

14. Derselbe, Klinik der Gelenkkrankheiten. 2. Aufl. I. Bd. 1876.

15. Derselbe, Verhandlungen der dentsch. Gesellschaft für Chir. VII. 1878. S. 107.

16. Hueter, H., Synovitis granul. hyperplast. Diese Zeitschrift XI. 317. 1879.

17. K ös ter, Ueber fungöse Gelenkentzündungen. Virchow's Arch. 48. S. 95. 1869.

18. Klebs, E., Virchow's Archiv. Bd. 44. S. 242. 1868. Ueber die erste Entstehung der Tuberkel u.s.w.

19. Koch, Aetiologie der Tuberculose. Berliner klin. Wocheuschrift. 1882. Nr. 15.

20. Ko cher, Th., Prophylaxis der fungösen Gelenkentzündung. Volkmann's klin. Vorträge. Nr. 102. 1876.

21. Kö nig, Fr., Befund miliarer Knötchen in fungösen Gelenken. Berliner klin. Wochenschrift. 1871. Nr. 20.

22. Derselbe, Tuberculose der Gelenke. Diese Zeitschrift XI. 531. 1879.

23. Derselbe, Die Erfolge der Resectionen u. s. w. Langenbeck's Archiv. XXV. S. 580.1880 .

24. Derselbe, Die Frühresection u. s. w. Verhandl. der d. Ges. für Chir. X. 93. 1881.

25. Derselbe, Die Tuberculose der Knochen und Gelenke. Volkmann's klin. Vorträge. $\mathrm{Nr}$. 214. 1882.

26. Lossen, Allgemeines über Resectionen. Pitha-Billroth's Handbuch der Chir. Bd. II. 2. Lief. 4. $\$ \$ 37-46.1881$.

27. Lü cke, Aetiologie der chronischen Ostitis. Diese Zeitschrift XIII. S. 300. 1880.

28. M in ch, Cariöse Erkrankungen des Fussskelets. Ebenda. XI. S. 317. 1879. 
29. Richet, Mém. sur les tumeurs blanches. Mém. de l'acad. T. 17. 1853.

30. Rinne, Centralblatt für Chirurgie. 1877.

31. Rust, Arthrokakologie. 181 .

32. Rokitansky, Lehrbuch der pathologischen Anatomie. II. S. 343. 1844.

33. Schede, M., Allgemeines über Amputationen. Pitha-Billroth, II. 2. Lief. 3. § 25. S. 37. 1881.

34. Derselbe, Ueber partielle Fussamputationen. Volkmann's klin. Vortr. Nr. $72 / 73$.

35. Sch üller, Centralblatt für Chirurgie. 1878. Nr. 43. 1879. Nr. 19.

36. Derselbe, Scrophulöse und tuberculöse Gelenkleiden. 1880.

36a. Derselbe, Die Aetiologie der chronischen Knochen- und Gelenkentzündungen. Diese Zeitschrift. XIV. S. 385, 1881.

37. Scriba, Berliner klin. Wochenschrift. 1877. Nr. 32.

38. Sonnenburg, Bedeutung der Tuberkel u.s. w. Verhandlungen der deutschen Gesellschaft für Chirurgie. X. S. 60. 1881.!

39. Talma, Ueber Virus tuberculos. D. Zeitschrift für klin. Med. II. S. 265. 1881. 40. Virchow, Archiv. Bd. IV. S. 312.

41. Derselbe, Geschwülste. II. S. 583, 652, 702, 706, 716-718, 1864.

42. Volkmann, R., Krankheiten der Bewegungsorgane. Pitha-Billroth, Bd. II. 2. Cap. 42. 1865-1872.

43. Derselbe, Charakter u. s. w. der fungösen Gelenkentzündung. Seine Vorträge. Nr. 168. 1879.

44. Wis eman, Several chirurg. treatises. L. IV. 1734.

45. Ziegler, E., Tuberculose u. Schwindsucht. Volkmam's kl.Vortr. Nr. 151. 1878.

46. Weigert, Zur Lehre von der Tuberculose. Virch. Arch. Bd. 77. S. 269.1879.

47. S ch ottelius, Zur Kritik der Tuberculosefrage I. Virchow's Arch. Bd. 91.1883.

48. Dettweiler u. Meissen, Der Tuberkelbacillus und die chronische Lungenschwindsucht. Berliner klin. Wochenschrift. 1883. Nr. 6 u. 7.

49. Heiberg, Die Tuberculose in ihrer anatom. Ausbreitung. Leipzig, Vogel. 1882. 50. Spina, A., Studien über Tuberculose. Wien, Braumuller. 1883.

51. Zieh l, Studien über den Tuberkelbacillus. D. med. Wochenschrift. 1883. Nr. 5 .

52. Koch, R., Erwiderung auf 47 u.s. w. D. med. Wochenschrift. 1883. Nr. 10. 